\title{
Corrigendum
}

\section{Ferreting things out: Biosecurity, pandemic flu and the transformation of experimental systems}

\author{
Natalie Hannah Porter
}

BioSocieties (2016) 11, 275. doi:10.1057/biosoc.2015.43; published online 26 October 2015

Correction to: BioSocieties, advance online publication 30 March 2015; doi:10.1057/ biosoc. 2015.4

The acknowledgment section was originally missing essential funding information and should have appeared as follows:

The research on which this original material is based has been subject to ethical review at the University of Oxford. The author does not have any competing intellectual or financial interests in the research detailed in the manuscript. The research for this article was provided by the European Research Council under the European Community's Seventh Framework Programme (FPT/2007-2013/ERC grant agreement no. 263447 (BioProperty). The author would like to thank her colleagues at the Institute for Science, Innovation, and Society and the Institute of Social and Cultural Anthropology at the University of Oxford for their insightful comments on this article. The author also gratefully acknowledges the critical commentary provided by Amy Hinterberger, Sabina Leonelli, Javier Lezaun, Catherine Montgomery, Kaushik Sunder Rajan and the anonymous reviewers at BioSocieties. A special thanks is due to the numerous participants and interlocutors of this research in the United States, the United Kingdom and Vietnam, who generously shared their time, facilities, resources and insights to inform this article.

The omission has now been rectified in the corrected version of the article. 